\title{
A Novel Defensin-Like Peptide Associated with Two Other New Cationic Antimicrobial Peptides in Transcriptome of the Iranian Scorpion Venom
}

\author{
Masoumeh Baradaran ${ }^{1}$, Amir Jalali ${ }^{2}$, Maryam Naderi soorki ${ }^{3}$ and Hamid Galehdari ${ }^{* 1}$ \\ ${ }^{1}$ Toxicology Research Center, Ahvaz Jundishapur University of Medical Sciences, Ahvaz, Iran; ${ }^{2}$ Department of \\ Pharmacology and Toxicology, Toxicology Research Center, Ahvaz Jundishapur University of Medical Sciences, Ahvaz, \\ Iran; ${ }^{3}$ Department of Genetics, Faculty of Sciences, Shahid Chamran University of Ahvaz, Ahvaz, Iran
}

Received 21 June 2016; revised 27 June 2016; accepted 2 July 2016

\begin{abstract}
Introduction: Scorpion venom is a source of bioactive peptides, and some antimicrobial peptides (AMPs) have been found in the venom gland of scorpions. Therefore, the discovery of new anti-infective agents is an essential need to overcome the problem of antibiotic resistance of clinical isolates. Here, we describe three new cationic AMPs, including meuVAP-6, meuAP-18-1, and meuPep34 from the venom gland of the Iranian scorpion, Mesobuthus eupeus. Methods: The cDNA sequences encoding all the three peptides were obtained from the cDNA library of scorpion venom gland and were deposited in the GenBank database. Results: MeuVAP- 6 and meuAP-18-1 are non-disulphide-bridged antimicrobial peptides, while meuPep34 is a cysteine-rich defensin-like peptide. Discussion: All three identified AMPs are rich in arginine and tryptophan. The overall results from the length, net charge, and hydrophobicity index suggested that meuPep34 could be the most active AMPs with the potential ability of biofilm inhibition. The data from molecular characterization of identified AMPs can provide a platform with application in drug discovery programs. DOI: 10.18869/acadpub.ibj.21.3.190
\end{abstract}

Keywords: Antimicrobial cationic peptides, Defensin-like peptide, Mesobuthus eupeus

Corresponding Author: Hamid Galehdari

Toxicology Research Center, Ahvaz Jundishapur University of Medical Sciences, Ahvaz, Iran; Tel.: (+98-613) 3338965;

E-mail: galehdari187@yahoo.com

\section{INTRODUCTION}

$\mathrm{D}$ efensins are a group of antimicrobial peptides (AMPs) and a part of innate immunity in vertebrates and invertebrates ${ }^{[1]}$. The first scorpion defensin was isolated from the hemolymph of the North African scorpion, Leiurus quinquestriatus, based on the similarity with insect defensin. It was also active against Gram-positive M. luteus but inactive against Gram-negative E. $\operatorname{coli}^{[2]}$. Later, some other AMPs with different effects were identified from various scorpion venomes ${ }^{[3]}$.

AMPs as a part of the immunity system of all animals have the length of four to more than 100 amino acids and also are wide-spectrum molecules ${ }^{[4]}$. Finding new effective AMPs is valuable because resistance to available antibiotics is rising constantly, and the need for new antibiotics to replace is increasing ${ }^{[5,6]}$. One promising class of antibiotics is AMPS $^{[7]}$. In the current study, we investigated the transcriptome of the Iranian scorpion Mesobuthus eupeus venom gland with the aim of finding new AMPs. This study represents the molecular and bioinformatics analysis of three founded AMPs.

\section{MATERIAS AND METHODS}

Double-stranded cDNA was synthesized from the total RNA extracted from the venom glands of $M$. eupeus. Following the cDNA cloning in appropriate cloning vector, the recombinant vectors were transformed into the bacterial host to prepare the cDNA library. The cDNA library was then sequenced 
and analyzed to find the AMPs. Nucleotide BLAST and protein BLAST were carried out using online BLAST tool in NCBI (blast.ncbi.nlm.nih.gov), while open reading frames (ORFs) were detected using the online ORF finder tool (www.ncbi.nlm.nih.gov/ orffinder/). Molecular weight, theoretical isoelectric $\mathrm{pH}$, and the net charge of peptides (at neutral $\mathrm{pH}$ ) were predicted using peptide property calculator online tool at Innovagen (www.innovagen.com/ proteomicstoo/ls). SignalP4.1 (http://www.cbs.dtu.dk/ services/ SignalP/) was also used to identify any signal peptide (http://www.cbs.dtu.dk/ services/SignalP/). The hydrophobicity of each peptide was calculated by GPMAW lite online tool available in http://www.alphalyse.com/ gpmaw_lite.html. The secondary structure of peptides was predicted by using the GOR secondary structure prediction method software (npsa-prabi.ibcp.fr/cgibin/npsa_automat.pl? page=npsa_gor4.html $)^{[8]}$. The alignment of every peptide was performed using Alignment tool in UniProt (www.uniprot.org/align). Transmembrane domains were predicted by the TMHMM tool (http://www.cbs.dtu.dk/services/
TMHMM/). The schematic diagrams of protein domain structures were drawn by using the Illustrator for biological sequences (IBS, version 1.0) software (http://ibs.biocuckoo.org/).

\section{RESULTS}

BLASTn analysis and protein BLAST identified four transcripts with acceptable similarity to the known scorpion AMPs $\left(E<10^{-3}\right)$. One of the transcripts namely CLAP has previously been identified and described by our team and deposited in NCBI with the accession number of $\mathrm{KC} 108907^{[9]}$. Other transcripts were named meuVAP-6, meuAP-18-1, and meuPep34. As shown in Figure 1, the cDNA sequences of all the three peptides have been deposited in GenBank under the accession numbers KU513845 (meuVAP-6), KU513846 (meuAP-18-1), and KU513849 (meuPep34). The full length cDNA of meuVAP-6 is $314 \mathrm{bp}$ that encodes a 70-amino acid peptide containing a 23-amino acid signal peptide, while that

(A)

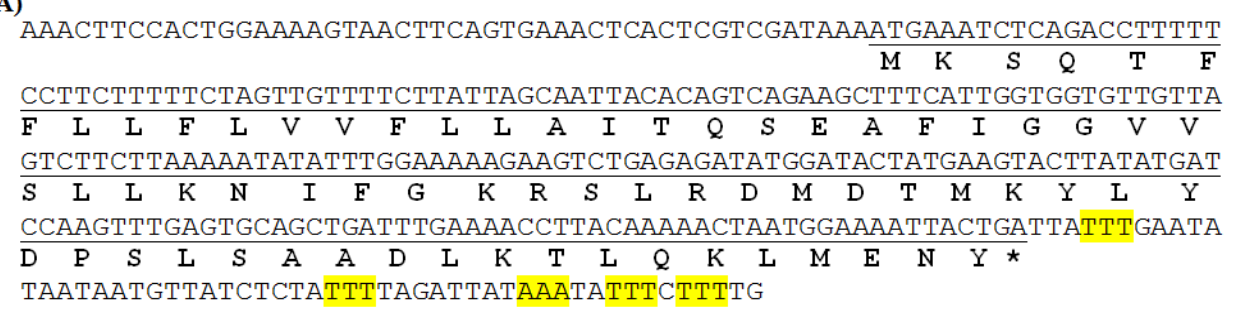

(B)

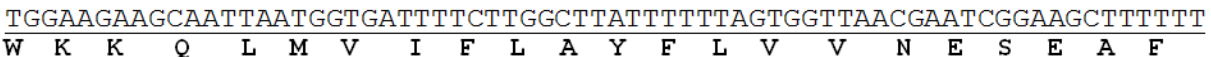
TGGGAGTCTTTTTAAATTAGCCACCAAGATAATTCCTAGTCTCTTCCGGAGGAAAAAGGAACGATCCA \begin{tabular}{lllllllllllllllllllllll}
\hline F & G & S & L & F & K & L & A & T & K & I & I & P & S & L & F & R & R & K & K & E & R & S
\end{tabular} TAATGAAAAGAGATTTGGAAAACCTATTCGACCCCTATCAACGCAATTTAGAAATGGATCGACTTTTG

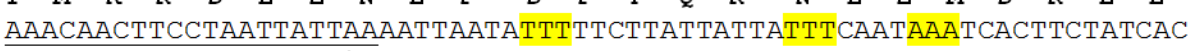

(C) K Q L P N Y *

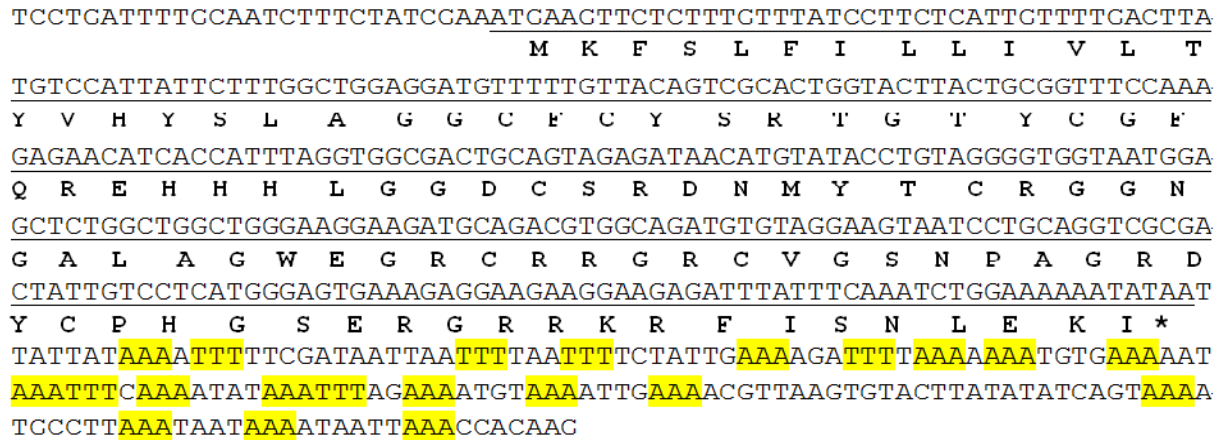

Fig. 1. cDNA and translated open-reading frame (ORF)for meuVAP-6 (A), meuAP-18-1 (B), and meuPep34 (C). Nucleotides of ORF are single underlined, and stop codons are indicated by asterisks. AAA and TTT motifs in 3'UTR are highlighted with yellow color. 
of meuAP-18-1 is 339 bp encoding a 76-amino acid peptide consisting of a 25 -amino acid signal peptide. The full length cDNA of 508 bp encoding a 103-amino acid peptide, meuPep34, was also found, with a 20 amino acid predicted signal.

The amino acid sequence alignments of meuVAP-6, meuAP-18-1, and meuPep34 with homologues in the UniProt database and the schematic image of peptides are indicated in Figures 2A, 2B, and 2C, respectively. The comparative sequence analysis of meuVAP-6 and meuAP-18-1 with other scorpion peptides revealed that both of them have some similarities with scorpion venom AMPs. Therefore, it can be concluded that meuVAP-6 and meuAP-18-1 are AMPs. The protein BLAST search and the alignment of meuPep34 with its counterparts indicated that meuPep34 is a peptide from scorpion as it is highly similar to AbCp-7 from scorpion Androctonus bicolor. The three-dimensional structure modeling of meuPep34 (Fig. 3) showed that meuPep34 has structural similarity with Bubble, a defensin protein, from Penicillium brevicompactum ${ }^{[10]}$ with the coverage of $29 \%$ and the sequence DNA similarity of $41 \%$. As a result, meuPep34 is supposed to be a new scorpion defensin-like peptide. In the predicted three-dimensional model, meuPep34 has three $\beta$-sheet strands.

MeuVAP-6 and meuAP-18-1 have no cysteine residue; therefore, they are classified in nondisulphide-bridged antimicrobial peptides (NDBPs), while meuPep34 contains 8 cysteine residues in which the pattern of $\mathrm{CXCX}_{7} \mathrm{CX}_{12} \mathrm{CX}_{7} \mathrm{CX}_{13} \mathrm{CX}_{4} \mathrm{CX}_{10} \mathrm{C}$ was found.

Predicted secondary structures and some physiochemical properties of meuVAP-6, meuAP-181 , and meuPep34 are summarized in Table 1. Based on the positive net charges and the existence of basic amino acids (arginine and tryptophan [RW]) among the amino acids of all the three peptides (Fig. 2A, 2B, and 2C), we concluded that the described peptides are all entirely cationic.

\section{DISCUSSION}

Scorpion venom gland is considered as a source of novel AMPs ${ }^{[3]}$. Considering the importance of AMPs as new anti-infective compounds, this study was designed to identify some novel anti-microbial peptides expressed in scorpion venom gland. In the current study, we presented three new cationic AMPs, including meuVAP-6, meuAP-18-1, and meuPep34.

Protein blasts of meuVAP-6 and meuAP-18-1 against homologues in the UniProt database indicates that $\mathrm{N}$-terminal and $\mathrm{C}$-terminal of both peptides are most highly conserved (Fig. 2a and 2B). Meucin-13 and Meucin-18-1 from Chinese $M$. eupeus with the identity of $91.4 \%$ and $92.1 \%$ are the most similar peptides to meuVAP-6 (Fig. 2A) and meuAP-18-1 (Fig. 2B), respectively. Regarding these similarities, it can be suggested that meuVAP-6 and meuAP-18-1 are the new variants of known peptides in the Chinese scorpion, or they are completly novel peptides. Moreover, the finding of identical peptides in the venom of different scorpion species has been indicated to be common ${ }^{[11]}$. Furthermore, the finding of similar peptides in scorpions from the same strain might be due to subspecies.

As mentioned above, meuVAP-6 and meuAP-18-1 are NDBPs. Zeng et al. ${ }^{[12]}$ have classified the scorpion NDBPs into six subfamilies. Based on this classification, meuVAP-6 and meuAP-18-1 are clustered in third subfamily containing long chain peptides, including hadrurin, parabutoporin, BmKbpp, pandinin 1, opistoporin 1, and opistoporin 2. In NDBPs grouping of Harrison et al. ${ }^{[3]}$, meuVAP-6 and meuAP18-1 are also placed in the long-chain peptide group. Zeng et al. ${ }^{[12]}$ have described some features for cDNA of NDBPs and their encoded precursors. These features are extended for meuVAP-6 and meuAP-18-1 and includes AAA or TTT motif at 3'UTR of cDNA (Fig. 1 ), the existence of a small neutral residue (glycine, alanine, or serine) at the cleavage site of signal peptide, the presence of one positively charged residue, Lys, in $\mathrm{N}$-terminal region, as well as one negatively charged residue (glutamic acid or aspartic acid) at $\mathrm{C}$-terminal region of each precursor (Fig. $2 \mathrm{~A}$ and $2 \mathrm{~B}$ ). It has been claimed that the presence of a rich AAA or TTT motif at 3'UTR of cDNA have positive effect on the stability of $\mathrm{RNA}^{[12]}$. Although meuPep34 is not a NDBP, it is very rich in AAA or TTT motif at 3'UTR of its cDNA (Fig. 1), which may affect the RNA stability.

Based on the existence of signal peptide ${ }^{[13]}$, it is suggested that all of the three described peptides are secretory peptides, i.e. when they are inserted into endoplasmic reticulum, the signal peptides are removed by a signal peptidase enzyme, and the mature peptide would be released in endoplasmic reticulum. Their transmembrane predicted domains (Fig. 2A, 2B, and $2 \mathrm{C}$ ) consist of 23 amino acids and contain a large part of signal peptide plus four or six (six in meuVAP6 and meuPep34, four in meuAP-18-1) beginning amino acids of mature peptides. It is proposed that the peptides attach to the membrane of endoplasmic reticulum through these domains.

It is known that the insertion of highly basic peptides into the cytosol perturb the integrity of the 
(A)

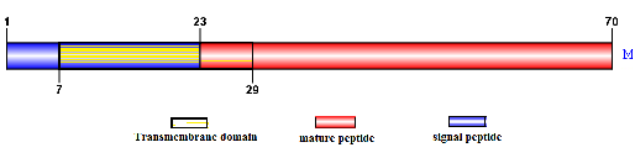
MKSQTFELLFLVYFLLAITQSEAFIGGVVSLLKNIFGKRSLRDMDTMKYLYDESLSAADLKTLQKLMENY

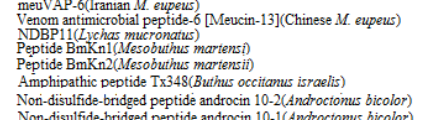
Noni-disulfide-bridged peptide androcin $10-2$ (Androctomus bicolor)
Non-disulfide-bridged peptide androcin 10-1 (Androctonus bicolor)
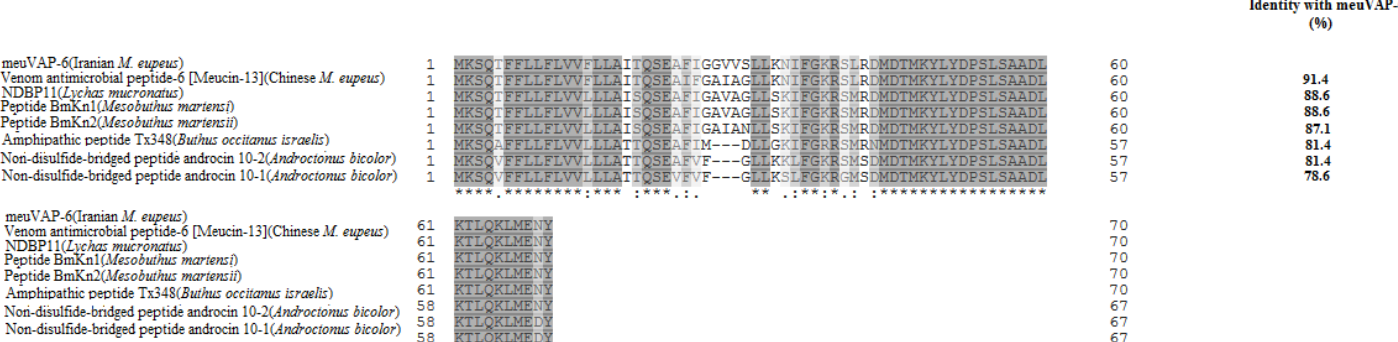

(B)

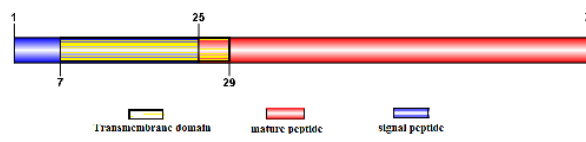

16
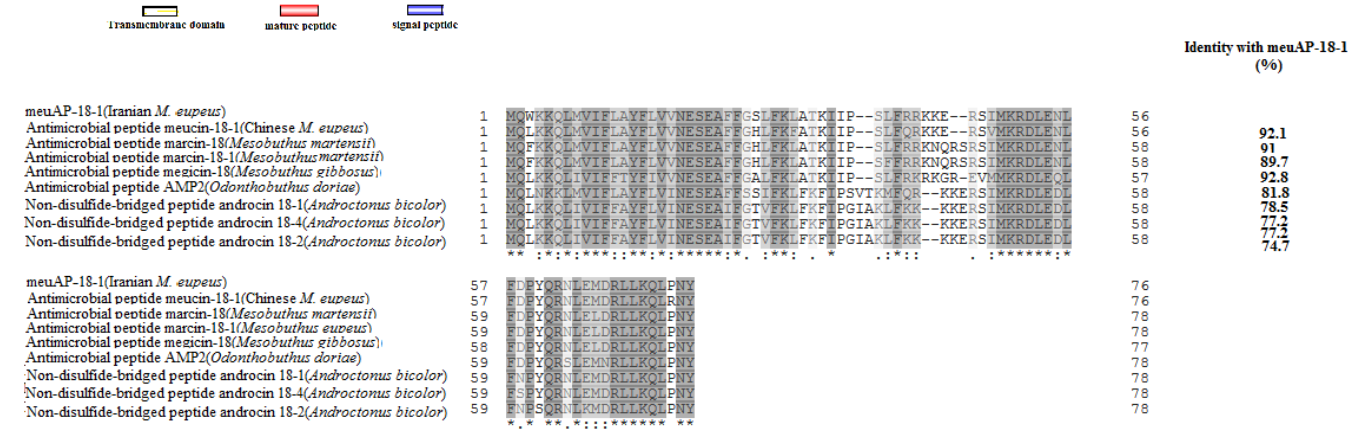

(C)
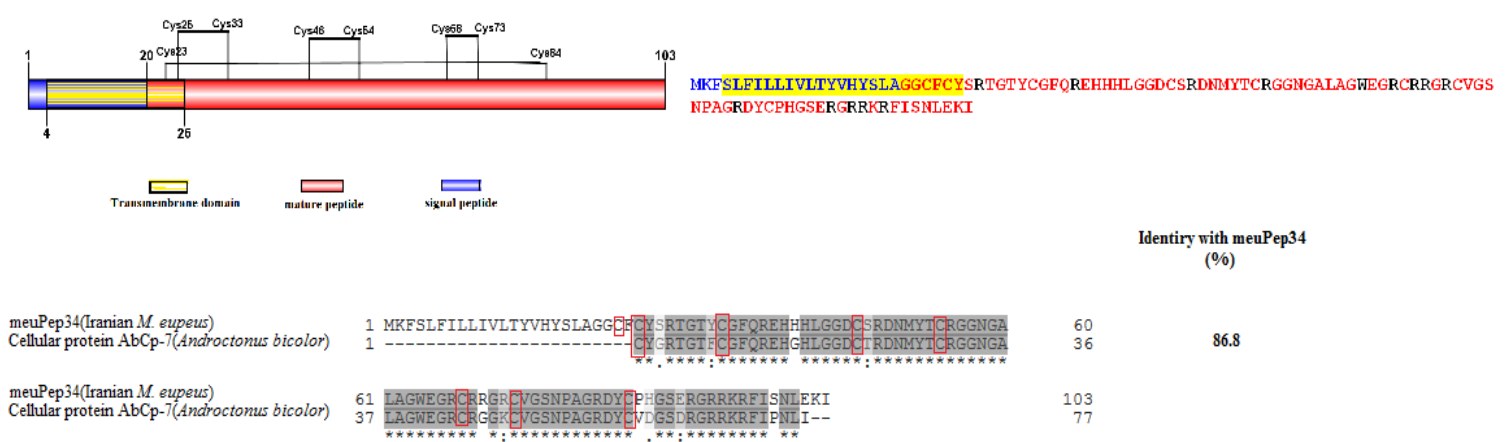

Fig. 2 The left images are schematic image of (A) meuVAP-6, (B) meuAP-18-1, and (C) meuPep34 with its domains. The right images showes aa sequences of (A) meuVAP-6, (B) meuAP-18-1, (C) meuPep34 and aa of each domain. (A) Down image indicates aa sequences alignment of meuVAP-6 with homologues, including venom antimicrobial peptide-6 (Meucin-13), Chinese M. eupeus (AC. no.: E4VP07), NDBP11, Lychas mucronatus (AC. no.: A0A0U1S7U5), Peptide BmKn1, Mesobuthus martensii (AC. no.: Q9GQW4), Peptide BmKn2, Mesobuthus martensii (AC. no.: Q6JQN2), Amphipathic peptide Tx348, Buthus occitanus israelis (AC. no.: B8XH50), non-disulfide-bridged peptide androcin 10-2, Androctonus bicolor (AC. no.: A0A0K0LBS0), non-disulfide-bridged peptide androcin 10-1, and Androctonus bicolor (AC. no.: A0A0K0LCH4). (B) Down image shows the aa sequences alignment of meuAP-181 with homologues, including antimicrobial peptide meucin-18-1, Chinese M. eupeus (AC. no.: F6K5S5), antimicrobial peptide marcin-18, Mesobuthus martensii (AC. no.: F6K5S6), antimicrobial peptide marcin-18-1, Mesobuthus martensii (AC. no.: F6K5S7), antimicrobial peptide megicin-18, Mesobuthus gibbosus (AC. no.: A0A059U8Y9), antimicrobial peptide AMP2, Odontobuthus doriae (AC. no: A0A0U4LVY4), non-disulfide-bridged peptide androcin 18-1, Androctonus bicolor (AC. no.: A0A0K0LBT6), non-disulfidebridged peptide androcin 18-4, Androctonus bicolor (AC. no.: A0A0K0LBT1), non-disulfide-bridged peptide androcin 18-2, Androctonus bicolor (AC. no.: A0A0K0LBT8). (C) Down image displys aa sequences alignment of meuPep34 with homologue peptide cellular protein AbCp-7 from Androctonus bicolor (Ac.no.: A0A0K0LC44). Conserved residues are represented in dark gray. Arginine and tryptophan are indicated with black color. Transmembrane domain is highlighted with yellow highlight. Residues with similar physicochemical properties are shown in light gray. Dash showes that there is not any residue in that position. The percentages of similarity with meuVAP-6, meuAP-18-1, and meuPep34 are brought in the right of alignment. Cystein residues are indicated with red rectangule. 


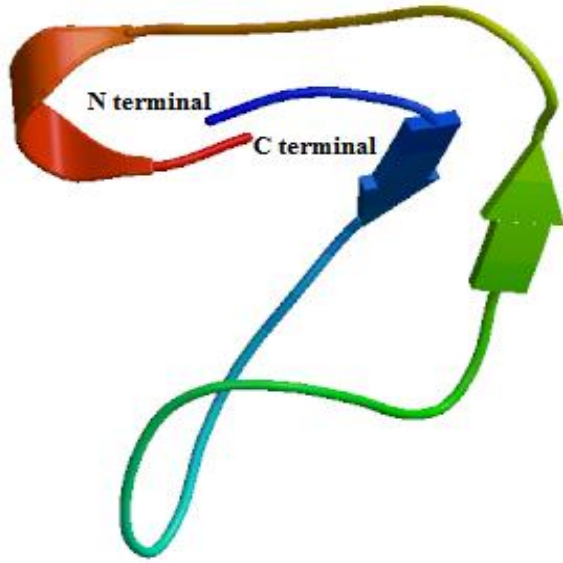

Fig. 3. A three-dimensional model of meuPep34

lipid bilaye ${ }^{[14]}$. On the other hand, the first and crucial step in the mode of AMPs action is interaction with microbial cells, which causes membrane perturbation. Disruption of the membrane, which is followed by some events such as cell wall biosynthesis or cell division, and/or translocation across the membrane to interact with cytoplasmic target, is leading to cell death $^{[15]}$. It is assumed that the positively charged amino acids of AMP initially interact with the negatively charged lipid head groups of the outer surface of the cytoplasmic membrane, which leads to bringing AMP near to the cytoplasm membrane ${ }^{[15,16]}$. Therefore, it can be concluded that a peptide with more positive net charge could be more potent against the microbial cells. In addition to electrostatic interaction, hydrophobocity is another item affecting AMP activity ${ }^{[17,18]}$. After close proximity to a lipid bilayer by the electrostatic interactions, AMPs can interact with cell membrane through hydrophobic forces ${ }^{[19-21]}$. In a research investigating the activity and the properties of analogs of BmKn1 from the venom of scorpion Buthus martensii Karsch, it has been observed that higher AMP hydrophobicity can be translated into more antimicrobial activity ${ }^{[22]}$. The comparative analysis of the present study described AMPs based on estimated net charges (meuVAP-6<meuAP-18-1<meuPep34), and hydrophobicity index (meuVAP-6>meuAP-18$1>$ meuPep34) implies that the most positively charged peptide has less hydrophobicity index. However, hydrophobic interactions in comparison to electrostatic forces are not long-ranged ${ }^{[23]}$, and it is suggested that the meuPep34 has more and the meuVAP-6 has less AMP activity.

A class of AMPs consisting of MeuVAP-6 has just $2 \mathrm{R}$, while meuPep34 has $13 \mathrm{R}$ and $1 \mathrm{~W}$, and meuAP-181 has $6 \mathrm{R}$ and $1 \mathrm{~W}$. In a study by Liu et al. ${ }^{[24]}$, the effect of chain length on the antimicrobial activity of RWrich AMPs was evaluated with synthesis of AMPs series with difference in the size of RW repeat. The results showed that the length of RW has a linear relationship with antimicrobial activity, i.e. the antimicrobial activity will increase with the elevation of RW length. Therefore, this result can be considered as a further evidence for the antimicrobial potency of meuPep34. Other study revealed that extracellular matrix are not able to affect biofilms ${ }^{[25]}$ although some antimicrobial peptides, which exert their function through the formation of diffusion barrier of RW-rich AMPs, can inhibit the biofilm growth of $E$. coli in a certain $\operatorname{dos}^{[26]}$. Hou et al. ${ }^{[27]}$ demonstrated that the chain length of RW-rich AMPs is important for the inhibition of bacterial growth in $E$. coli biofilm since AMPs with more RW are more potent. This fact improves the possibility that meuPep34 having $13 \mathrm{R}$

Table 1. Predicted Secondary structure and some physiochemical properties of peptides described in this study

\begin{tabular}{|c|c|c|c|c|c|}
\hline $\begin{array}{l}\text { Peptide } \\
\text { name }\end{array}$ & Secondary structure & $\begin{array}{c}\text { Molecular } \\
\text { weight }\end{array}$ & $\begin{array}{c}\text { Isoelectric } \\
\text { pI }\end{array}$ & $\begin{array}{l}\text { Hidrophobicity } \\
\text { index }\end{array}$ & $\begin{array}{c}\text { Net charge } \\
\text { at pH } 7\end{array}$ \\
\hline meuVAP-6 & 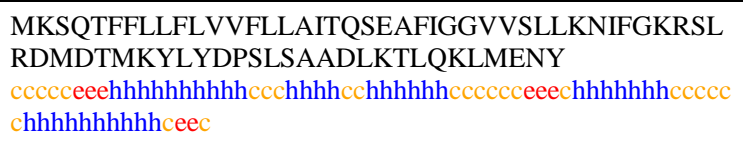 & 8014.45 & 9.61 & 0.37 & 2.0 \\
\hline meuAP-18-1 & 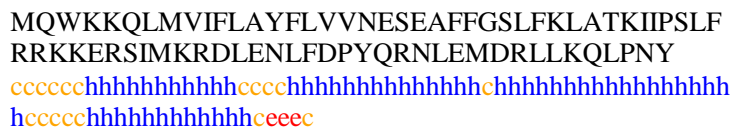 & 9289.96 & 10.42 & -0.25 & 6.0 \\
\hline meuPep34 & 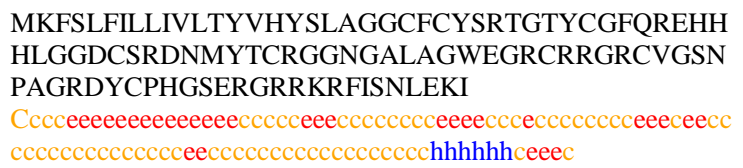 & 11614.19 & 9.28 & -0.47 & 8.9 \\
\hline
\end{tabular}

The random coil is represented by "c", the extended strand by "e" and the helix by " $h$ " 
and $1 \mathrm{~W}$ could be the potent inhibitor of biofilms. Moreover, as it can be conferred from the amino acid alignment (Fig. 2C), just one uncharacterized scorpion homologue exists for meuPep34. However, based on structural modeling that indicated a similarity with a known defensin, we suggest that meuPep34 is possibly a defensin. The alignment of meuPep34 with its homologue revealed that unlike the meuPep34, its homologue has seven cysteine residues, which is one residue less than meuPep34. The difference in numbers of cysteines may affect the final structure of the peptide. In the present study, we have identified new peptides in the Iranian Scorpion Venom with putative antibacterial activity. Therefore, the result of this study may introduce novel antimicrobial peptides to pharmaceutical industry.

\section{ACKNOWLEDGMENTS}

This study was financially supported by Ahvaz Jundishapur University of Medical Sciences (Ahvaz, Iran). We are thankful of Shahid Chamran University authorities for assistance in preparing laboratory equipments. We also thank Dr. Babak Vazirian for his kind supports.

\section{CONFLICT OF INTEREST. None declared.}

\section{REFERENCES}

1. Ganz T. Defensins: antimicrobial peptides of innate immunity. Nature reviews immunology 2003; 3(9):710720.

2. Bontems F, Roumestand C, Gilquin B, Menez A and Toma F. Refined structure of charybdotoxin: common motifs in scorpion toxins and insect defensins. Science 1991; 254(5037): 1521-1523.

3. Harrison PL, Abdel-Rahman MA, Miller K, Strong PN. Antimicrobial peptides from scorpion venoms. Toxicon 2014; 88: 115-137.

4. Wenzel M, Chiriac AI, Otto A, Zweytick D, May C, Schumacher C, Gust R, Albada HB, Penkova M, Kramer U, Erdmann R, Metzler-Nolte N, Straus SK, Bremer E, Becher D, Brotz-Oesterhelt H, Sahl HG and Bandow JE. Small cationic antimicrobial peptides delocalize peripheral membrane proteins. Proceedings of the national academy of sciences USA 2014; 111(14): E1409-E1418.

5. Tavares LS, Silva CSF, de Souza VC, da Silva VL, Diniz CG and Santos MO. Strategies and molecular tools to fight antimicrobial resistance: resistome, transcriptome, and antimicrobial peptides. Frontiers in microbiology 2013; 4: 412 .

6. Bahar AA and Ren D. Antimicrobial Peptides.
Pharmaceuticals 2013; 6(12): 1543-1575.

7. Malmsten M. Antimicrobial peptides. Upsala journal of medical sciences 2014; 119(2): 199-204.

8. Garnier J, Gibrat JF, Robson B. GOR method for predicting protein secondary structure from amino acid sequence. Methods in enzymology 1996; 266: 540-553.

9. Baradaran M, Jalali A, Jolodar A, Ghasemian S. New caerin-like antibacterial peptide from the venom gland of the Iranian scorpion Mesobuthus eupeus: cDNA amplification and sequence analysis. African journal of biotechnology 2012; 11: 10176-10181.

10. Olsen JG, Flensburg C, Olsen O, Bricogne G, Henriksen A. Solving the structure of the bubble protein using the anomalous sulfur signal from single-crystal in-house $\mathrm{Cu}$ Kalpha diffraction data only. Acta crystallographica section biological crystallography 2004; 60(Pt 2): 250255.

11. Kuzmenkov AI, Vassilevski AA, Kudryashova KS, Nekrasova OV, Peigneur S, Tytgat J, Feofanov AV, Kirpichnikov MP, Grishin EV. Variability of potassium channel blockers in mesobuthus eupeus scorpion venom with focus on Kv1.1: An integrated transcriptomic and proteomic study. Journal of biological chemistry 2015; 290(19): 12195-12209.

12. Zeng XC, Corzo $G$ and Hahin R. Scorpion venom peptides without disulfide bridges. The international union of biochemistry and molecular biology life 2005; 57(1): 13-21.

13. Nothwehr SF, Gordon JI. Targeting of proteins into the eukaryotic secretory pathway: signal peptide structure/function relationships. Bioessays 1990; 12(10): 479-484.

14. Mabrouk K, Van Rietschoten J, Vives E, Darbon H, Rochat H, Sabatier JM. Lethal neurotoxicity in mice of the basic domains of HIV and SIV Rev proteins. Study of these regions by circular dichroism. FEBS letters 1991; 289(1): 13-17.

15. Fjell CD, Hiss JA, Hancock RE, Schneider G. Designing antimicrobial peptides: form follows function. Nature reviews drug discovery 2011; 11(1): 37-51.

16. Taheri-Araghi S, Ha B-Y. Cationic antimicrobial peptides: a physical basis for their selective membranedisrupting activity. Soft matter 2010; 6: 1933-1940.

17. Dathe M, Nikolenko H, Meyer J, Beyermann M, Bienert M. Optimization of the antimicrobial activity of magainin peptides by modification of charge. FEBS letters 2001; 501(2-3):146-150.

18. Taheri-Araghi S, Ha BY. Physical basis for membranecharge selectivity of cationic antimicrobial peptides. Physical review letters. 2007; 98(16): 168101.

19. Shai Y. Mechanism of the binding, insertion and destabilization of phospholipid bilayer membranes by alpha-helical antimicrobial and cell non-selective membrane-lytic peptides. Biochimica et biophysica acta 1999; 1462(1-2): 55-70.

20. Zasloff M. Antimicrobial peptides of multicellular organisms. Nature 2002; 415(6870): 389-395.

21. Brogden KA. Antimicrobial peptides: pore formers or metabolic inhibitors in bacteria? Nature reviews 
microbiology 2005; 3(3): 238-250.

22. de la Salud Bea R, Ascuitto MR, de Johnson LE. Synthesis of analogs of peptides from Buthus martensii scorpion venom with potential antibiotic activity. Peptides 2015; 68: 228-232.

23. Lee MT, Chen FY and Huang HW. Energetics of pore formation induced by membrane active peptides. Biochemistry 2004; 43(12): 3590-3599.

24. Liu Z, Brady A, Young A, Rasimick B, Chen K, Zhou C, Kallenbach NR. Length effects in antimicrobial peptides of the $(\mathrm{RW})_{\mathrm{n}}$ Series. Antimicrobial agents and chemotherapy 2007; 51(2): 597-603.
25. Lewis K. Riddle of biofilm resistance. Antimicrob agents chemotherapy 2001; 45(4): 999-1007

26. Hou S, Zhou C, Liu Z, Young AW, Shi Z, Ren D, Kallenbach NR. Antimicrobial dendrimer active against Escherichia coli biofilms. Bioorganic and medicinal chemistry letters 2009; 19(18): 5478-5481.

27. Hou S, Liu Z, Young AW, Mark SL, Kallenbach NR, Ren D. Effects of Trp- and Arg-containing antimicrobial-peptide structure on inhibition of Escherichia coli planktonic growth and biofilm formation. Applied and environmental microbiology 2010; 76(6): 1967-1974. 